\title{
Tandem cervical carotid stenting for stenosis with flow diversion embolisation for the treatment of intracranial aneurysms
}

\author{
Jessica K Campos, ${ }^{1}$ Li-Mei Lin, ${ }^{2}$ Narlin B Beaty, ${ }^{1}$ Matthew T Bender, ${ }^{1}$ \\ Bowen Jiang, ${ }^{1}$ David A Zarrin, ${ }^{1}$ Alexander L Coon ${ }^{1}$
}

To cite: Campos JK, Lin L-M, Beaty NB, et al. Tandem cervical carotid stenting for stenosis with flow diversion embolisation for the treatment of intracranial aneurysms. Stroke and Vascular Neurology 2019;4: e000187. doi:10.1136/svn-2018-000187

Received 18 August 2018 Revised 6 October 2018 Accepted 12 November 2018 Published Online First 17 December 2018

\section{Check for updates}

(C) Author(s) (or their employer(s)) 2019. Re-use permitted under CC BY-NC. No commercial re-use. See rights and permissions. Published by BMJ.

${ }^{1}$ Department of Neurosurgery, Johns Hopkins University School of Medicine, Baltimore, Maryland, USA

${ }^{2}$ Department of Neurosurgery, University of California, Irvine, Orange, California, USA

Correspondence to Dr Alexander L Coon; dralexandercoon@gmail.com

\section{ABSTRACT}

Background An estimated $2 \%-3 \%$ of the population harbour an intracranial aneurysm. Concomitant atherosclerotic cervical carotid disease is not uncommon. The management of these two entities remains a challenge within the field.

Case presentation We report a single case of concomitant carotid stenosis and two ipsilateral unruptured intracranial aneurysms treated with a single-staged cervical carotid stenting and cerebral aneurysm embolisation with the Pipeline embolisation device.

Discussion No consensus currently exists to guide endovascular treatment of intracranial aneurysms associated with asymptomatic ipsilateral stenosis. Here, we present a case of asymptomatic moderate carotid stenosis with two ipsilateral intracranial aneurysms and suggest carotid artery stenting takes procedural priority over aneurysm embolisation in single-stage treatment. The rationale for the sequence of neurointerventions is based on the tracking a robust distal access system beyond a stenotic proximal carotid lesion and stabilisation of the ulcerated plaque to avoid thromboembolic complications associated with plaque irritation during aneurysm embolisation. Additional cases and longer follow-up will be needed to further assess the efficacy of this technique.

\section{INTRODUCTION}

An estimated $2 \%-3 \%$ of the population harbour an intracranial aneurysm with some of these patients harbouring a concomitant cervical internal carotid artery stenosis. ${ }^{12}$ No consensus currently exists to guide endovascular treatment of intracranial aneurysms associated with asymptomatic ipsilateral extracranial carotid stenosis; however, case reports and small-volume case series have addressed treatment strategies for symptomatic extracranial carotid stenosis and concomitant incidental intracranial aneurysms. $^{2-5}$ More recent publications discuss treating both vascular pathologies in a staged or tandem approach and portend risks associated with the sequence of carotid revascularisation and aneurysm treatment.
As endovascular neurointerventions continue to emerge as the primary treatment modality for cerebral aneurysms, notably flow diversion embolisation, the technical aspects of single-stage tandem carotid artery stenting and aneurysm embolisation require further exploration. Here, we present a case and discuss the technical aspects of simultaneous carotid artery stenting and flow diversion aneurysm embolisation with the Pipeline embolisation device (PED; Medtronic Neurovascular, Irvine, California, USA) in a patient with concomitant carotid stenosis and ipsilateral, unruptured cerebral aneurysms.

\section{CASE PRESENTATION}

A patient presented to our institution with a duplex ultrasonography finding of asymptomatic $50 \%$ stenosis of the right carotid artery. The patient has a history of chronic obstructive pulmonary disease (COPD), congestive heart failure (CHF), status post aortic valve replacement for severe aortic stenosis, former smoker and two first-degree relatives with a history of aneurysmal subarachnoid haemorrhage. Magnetic resonance angiography revealed $50 \%$ stenosis of the right carotid artery, a $3 \mathrm{~mm}$ anterior choroidal aneurysm and a $5 \mathrm{~mm}$ communicating segment aneurysm. The aneurysms were followed with interval non-invasive imaging procedures for three consecutive years.

On successive scans, the anterior choroidal aneurysm increased in size to $4.2 \mathrm{~mm}$, and the patient was consented for a cerebral catheter angiogram. Angiography demonstrated $62 \%$ carotid stenosis with contrast stagnation behind the atherosclerotic plaque and an aneurysmal complex along the right intracranial carotid artery (ICA). The patient gave informed consent for single-staged carotid artery stenting (CAS) followed by flow diversion aneurysm embolisation with the PED. 


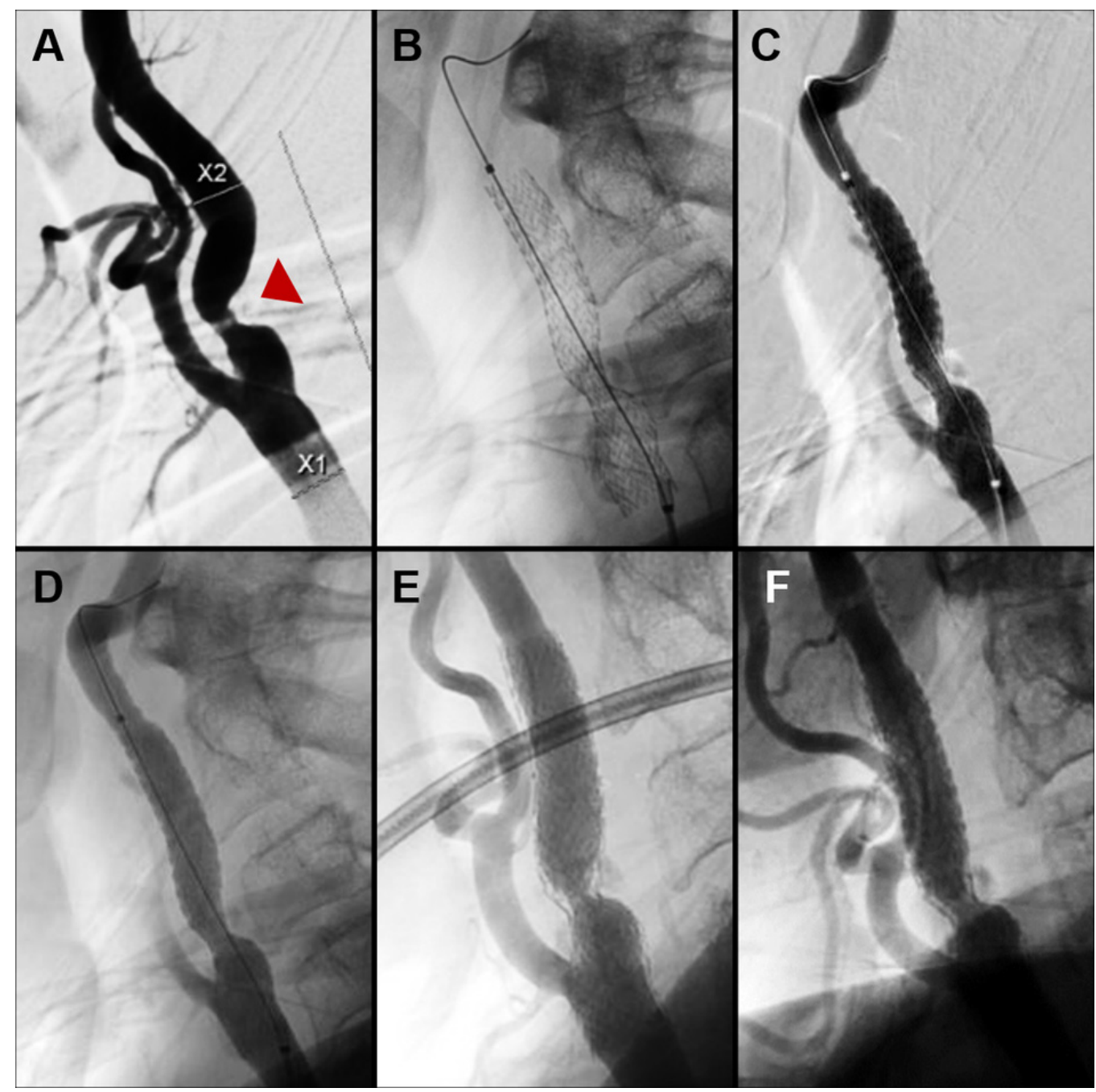

Figure 1 Carotid artery stent revascularisation treatment for cervical carotid stenosis. (A) Digital subtraction angiogram (lateral view) of right common carotid artery demonstrating stenosis distal to bifurcation (arrowhead). (B) Native fluoroscopy, lateral view, illustrating placement of self-expanding carotid stent across the length of stenotic lesion. Lateral view (C) control angiography and (D) native fluoroscopy immediately following stent placement confirms optimal luminal re-establishment. Follow-up at (E) 6 months and (F) 12 months demonstrates favourable luminal expansion after cervical carotid revascularisation.

Aspirin (325 mg/day) and clopidogrel (75 mg/day) were administered 7 days prior to the procedure.

The procedure was performed under single-agent conscious sedation and monitored by an anaesthesiologist. During the intervention, $5000 \mathrm{IU}$ of heparin was administered. An 8 Fr $90 \mathrm{~cm}$ AXS Infinity LS sheath (Stryker Neurovascular, Freemont, California, USA) was used for distal access within the right common carotid artery. Angiography demonstrated a preocclusive lesion at the proximal ICA (figure 1A). The stenotic lesion was traversed with a distal protection device (Spider FX, EV3), with predilatation from a $6 \mathrm{~mm}$ balloon catheter (Maverick PTCA balloon; Boston Scientific, Watertown, Massachusetts, USA), and a self-expanding $8 \mathrm{~mm}$ by $40 \mathrm{~mm}$ Protégé RX carotid stent (Medtronic, Minneapolis, Minnesota, USA) was implanted across the ulcerated plaque (figure 1B).

Control subtraction angiography demonstrated optimal luminal re-establishment of the right carotid artery (figure $1 \mathrm{C}-\mathrm{D}$ ) and again confirmed the presence of both ipsilateral aneurysms (figure 2A-B) .

Following carotid stent placement, the distal protection device was recaptured and the Pipeline triaxial catheter platform was navigated through the long sheath and through the carotid stent. The $90 \mathrm{~cm}$ sheath was then tracked through the carotid stent and placed within the ICA distal to the stent, to minimise friction of the triaxial system along the stent. A single $3.75 \mathrm{~mm}$ by $16 \mathrm{~mm}$ PED was deployed across the neck of both aneurysms (figure 2C-D). The patient tolerated the procedure without any neurological deficits. The patient was discharged from the hospital the following day on aspirin (325 mg/day) and clopidogrel (75 mg/ day).

Follow-up angiography revealed a widely patent carotid stent (figure 1E-F), and the aneurysms demonstrated no residual filling (figure $2 \mathrm{E}-\mathrm{F}$ ). The patient remained at neurological baseline at 6-month follow-up. Clopidogrel 


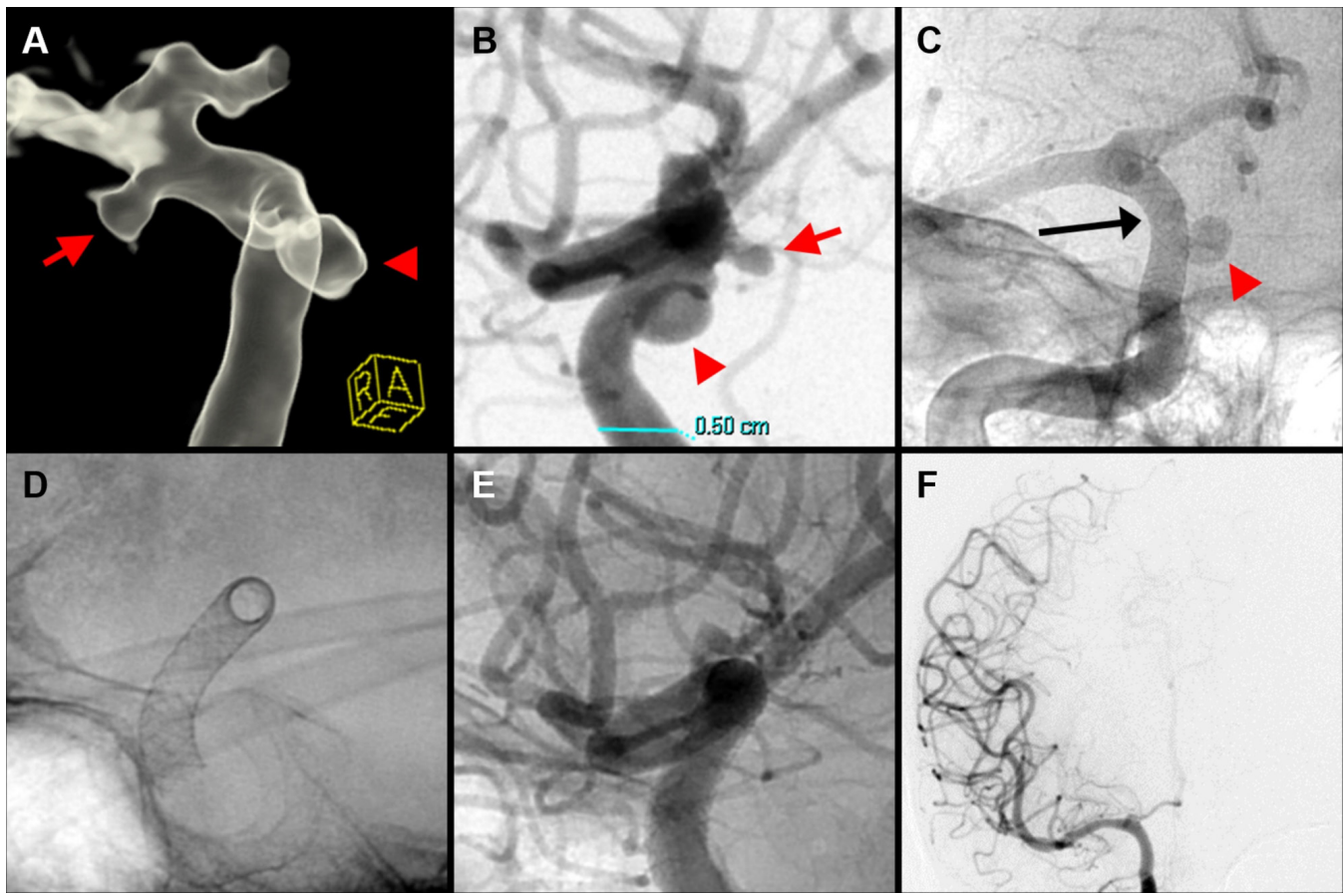

Figure 2 Pipeline embolisation device (PED) treatment of two right-sided intracranial carotid artery (ICA) aneurysms, 4 mm anterior choroidal aneurysm and a $5 \mathrm{~mm}$ communicating segment aneurysm, with occlusion demonstrated at $12 \mathrm{months}$. (A) Pre-embolisation 3D-rotational reconstructed image, oblique view, and (B) digital subtraction angiogram (lateral view) of right ICA demonstrating the right anterior choroidal aneurysm (red arrow) and communicating segment aneurysm (red arrowhead). Native fluoroscopy (C, anteroposterior (AP) view; D, lateral view) immediately following deployment confirms the single 3.75 mm by $16 \mathrm{~mm}$ PED (black arrow) was implanted across the length of both aneurysms with appropriate wall apposition (red arrow, communicating segment aneurysm). At 12 months, (E, lateral view) follow-up digital subtraction angiogram of the right ICA confirms occlusion of both aneurysms and (F, AP view) patent distal vasculature.

was discontinued after the 6-month angiogram and the patient continues with aspirin $325 \mathrm{mg}$ daily.

\section{DISCUSSION}

This is a single-case report of concomitant asymptomatic carotid stenosis and two ipsilateral unruptured intracranial aneurysms treated with a single-staged carotid stenting and aneurysm embolisation with the PED. No complications were observed periprocedurally following embolisation or over the initial 6 months of clinical follow-up. No consensus currently exists on the treatment paradigm for the endovascular treatment of intracranial aneurysms associated with asymptomatic ipsilateral stenosis.

Historically, the presence of concomitant vasculopathologies have been addressed with separate, staged treatments. Primary carotid stenting may increase the risk of ipsilateral aneurysm rupture from altered haemodynamics increasing forward flow towards the aneurysm and also presents the risk of carotid stent disturbance during successive aneurysm treatment. ${ }^{23}$ However, embolisation of an aneurysm through untreated carotid stenosis carries its own risks. The mechanical interaction between the access catheters and stenotic carotid plaque may increase the risk of thromboembolic complications, especially given the inability to place a distal protection device during aneurysm embolisation. While it is not known to what extent carotid stenosis protects an ipsilateral distal aneurysm via haemodynamic compromise, or how revascularisation of a stenotic lesion may aggravate aneurysm rupture, the current literature and theories support effectively addressing both lesions while minimising time between treatment of the concomitant diseases. ${ }^{3}$ As the safety of endovascular modalities for aneurysm treatment and carotid stenosis continues to improve, the notion of simultaneous single-staged treatment has increasing appeal. ${ }^{45}$ Small volumes of cases within the literature have demonstrated the feasibility of simultaneous singlestaged treatment of concomitant carotid stenosis and unruptured ipsilateral aneurysms. ${ }^{3-9}$ Reassuringly, it has been postulated that the additive risk of periprocedural complications in staged CAS and aneurysm embolisation can be consolidated in a single-stage procedure. ${ }^{8}$

Consideration was given to treatment of the carotid lesion with either traditional carotid endarterectomy (CEA) or endovascular CAS. A growing body of literature cites the non-inferiority of CAS with embolic protection compared with carotid endarterectomy in asymptomatic patients, and endovascular recanalisation of carotid artery stenosis has shown similar rates of treatment efficacy as compared with CEA. ${ }^{10-12}$ Patient-specific factors, including history of COPD and CHF status post aortic valve replacement for severe aortic stenosis, significantly increased the risk associated with open surgical treatment by CEA. ${ }^{13}$ Additionally, the need for a dual antiplatelet 
regimen following distal aneurysm embolisation with the PED was a deterrent to open surgical treatment.

Surgical planning for single-stage treatment of concomitant extracranial carotid stenosis and ipsilateral intracranial aneurysms must address of the sequence of neurointerventions with particular attention to the plaque morphology. ${ }^{7}$ Recent case reports and smallvolume case series suggest CAS takes precedent over aneurysm embolisation in the single-stage treatment of both concomitant vasculopathologies. ${ }^{6-9}$ However, historical theories suggest acute changes in cerebral perfusion pressures following carotid stenosis revascularisation may increase the rupture risk of untreated ipsilateral aneurysms, secondary to a theoretical increase in aneurysm wall shear stress. ${ }^{6}$ To date, no reports of periprocedural or delayed aneurysm rupture have been documented within published case series of single-staged CAS and coil embolisation of ipsilateral intracranial aneurysms. ${ }^{8914} \mathrm{In}$ this case, the rationale for the sequence of neurointerventions was based on the tracking a robust distal access system beyond a stenotic proximal carotid lesion and stabilisation of the ulcerated plaque to avoid thromboembolic complications associated with plaque irritation during aneurysm embolisation. These previous reports have been with traditional coiling techniques that necessitated microcatheter passage across the cervical stenosis and both microcatheters and intermediate catheters. Additional cases and longer follow-up will be needed to further assess the efficacy of this technique.

Endovascular treatment of asymptomatic moderate carotid stenosis is not typically performed. The rationale for CAS in this case, prior to aneurysm embolisation, was threefold. Namely, to allow adequate access of a robust intracranial triaxial access platform for the planned aneurysm flow diversion through a stenotic proximal cervical carotid lesion. Second, carotid stenting protected the ulcerative plaque against irritation from positioning of the long guide sheath and intermediate guide catheter. Furthermore, initial contact with the ulcerative plaque was made under the supervision of a distal protection device; this would not have been possible had the aneurysm been treated first. Securing the carotid stent across the plaque with a distal protection device minimised the risk of thromboembolic complications. In this report, the robust intracranial triaxial access platform used consisted of a distal intracranial catheter telescoped within a $6 \mathrm{Fr}$ $90 \mathrm{~cm}$ long guide sheath. An AXS Infinity LS guide sheath (Infinity; Stryker Neurovascular) was used and tracked to a final position within the high cervical ICA, promoting stability across the aortic arch and proximal carotid artery during PED deployment. ${ }^{15}$ To our third motive for CAS treatment, navigation of the $8 \mathrm{Fr}$ outer diameter Infinity long sheath across the point of maximal ICA stenosis, measuring $2.8 \mathrm{~mm}$, would otherwise result in $>90 \%$ obstruction of the vessel lumen. ${ }^{15}$ Ensuring anterograde flow around the robust access system within the cervical carotid presented compelling rationale for CAS prior to aneurysm treatment.
Care should be taken to both reduce the potential for carotid vessel damage and prevent migration of the carotid stent. Vessel damage can be mitigated by meticulous assessment of the carotid vasculature. The appropriately sized open-cell carotid stent, that is, final stent diameter, is selected to approximate both the diameter of the vessel just proximal and distal to the stenosis. The open-cell stent is able to generate larger expansive forces secondary to the larger free cell area of its design. ${ }^{16}$ The open-cell carotid stent design was chosen for its flexibility and ability to achieve superior wall apposition across the steep angulation of the stenotic plaque and minimise the risk of thrombotic complications. To prevent delayed device migration, we recommend ensuring the device is sufficiently opposed to the diseased vessel wall, using balloon dilation if necessary. Meticulous attention to navigating the long sheath and microcatheter for PED embolisation, under continuous fluoroscopy, is paramount to minimising disruption of the implanted carotid stent. Both the microcatheter and intermediate catheter should be tracked coaxially high into the distal carotid prior to navigating the long sheath through the carotid stent. We also recommended tracking the distal tip of the long sheath sufficiently beyond the distal margin of the carotid stent to avoid overlap of the intermediate catheter on the distal tines of the carotid stent. This protects the intermediate catheter from mechanical damage and aids in diagnostic quality cerebral contrast injections through the long sheath.

The primary limitation of this study is the single-case nature of the report. We present this as more of a feasibility report and demonstration of concept rather than clinical evidence. Safety and effectiveness of this simultaneous technique will need to be evaluated further through multicentre registries that can enrol enough patients to evaluate these procedures adequately.

\section{CONCLUSION}

We report a single case of concomitant carotid stenosis and two ipsilateral unruptured intracranial aneurysms treated safely with a single-staged carotid stenting and Pipeline aneurysm embolisation. Both interventions were performed successfully through a single vascular access site in a sequential fashion of carotid stenting with distal embolic protection followed by PED implantation. The cerebral aneurysm was completely occluded, and the plaque stabilised at last clinical follow-up 6 months after the procedure.

Contributors JKC, L-ML and NBB drafted the manuscript and critically revised the manuscript for important intellectual content. MTB, BJ and DAZ assisted with the data collection and analysis. ALC performed treatment procedures and critically revised the important intellectual content. All authors read and approved the final manuscript.

Funding The authors have not declared a specific grant for this research from any funding agency in the public, commercial or not-for-profit sectors.

Competing interests ALC is a consultant for InNeuroCo, a consultant and proctor for Medtronic Neurovascular, MicroVention-Terumo and Stryker Neurovascular. 
L-ML is a proctor for Medtronic Neurovascular. The other authors have no conflict of interest. No author received financial support in conjunction with the generation of this submission.

Patient consent for publication Not required.

Ethics approval Johns Hopkins Institutional Review Board.

Provenance and peer review Not commissioned; externally peer reviewed. Data sharing statement There are no additional unpublished data from this study. Open access This is an open access article distributed in accordance with the Creative Commons Attribution Non Commercial (CC BY-NC 4.0) license, which permits others to distribute, remix, adapt, build upon this work non-commercially, and license their derivative works on different terms, provided the original work is properly cited, appropriate credit is given, any changes made indicated, and the use is non-commercial. See: http://creativecommons.org/licenses/by-nc/4.0/.

\section{REFERENCES}

1. Kappelle LJ, Eliasziw M, Fox AJ, et al. Small, unruptured intracranial aneurysms and management of symptomatic carotid artery stenosis. North American Symptomatic Carotid Endarterectomy Trial Group. Neurology 2000;55:307-9.

2. Pappadà G, Fiori L, Marina R, et al. Incidence of asymptomatic berry aneurysms among patients undergoing carotid endarterectomy. $J$ Neurosurg Sci 1997;41:257-62.

3. Adams HP. Carotid stenosis and coexisting ipsilateral intracranial aneurysm. A problem in management. Arch Neurol 1977;34:515-6.

4. Becske T, Brinjikji W, Potts MB, et al. Long-term clinical and angiographic outcomes following pipeline embolization device treatment of complex internal carotid artery aneurysms: five-year results of the pipeline for uncoilable or failed aneurysms trial. Neurosurgery 2017;80:40-8.

5. Colby GP, Bender MT, Lin LM, et al. Declining complication rates with flow diversion of anterior circulation aneurysms after introduction of the Pipeline Flex: analysis of a single-institution series of 568 cases. J Neurosurg 2018;12:1-7.

6. Iwata T, Mori T, Tajiri H. Successful staged endovascular treatment of a symptomatic cervical carotid bifurcation stenosis coupled with a coincidental unruptured cerebral aneurysm in the carotid distal segment. AJNR Am J Neuroradiol 2008;29:1948-50.

7. Cvetic V, Dragas M, Colic M, et al. Simultaneous endovascular treatment of tandem internal carotid lesions: case report and review of literature. Vasc Endovascular Surg 2016;50:359-62.

8. Badruddin A, Teleb MS, Abraham MG, et al. Safety and feasibility of simultaneous ipsilateral proximal carotid artery stenting and cerebral aneurysm coiling. Front Neurol 2010;1:120.

9. Park JC, Kwon BJ, Kang HS, et al. Single-stage extracranial carotid artery stenting and intracranial aneurysm coiling: technical feasibility and clinical outcome. Interv Neuroradiol 2013;19:228-34.

10. Gurm HS, Yadav JS, Fayad P, et al. Long-term results of carotid stenting versus endarterectomy in high-risk patients. N Engl J Med 2008;358:1572-9.

11. Rosenfield K, Matsumura JS, Chaturvedi S, et al. Randomized trial of stent versus surgery for asymptomatic carotid stenosis. N Engl $J$ Med 2016;374:1011-20.

12. Brott TG, Howard G, Roubin GS, et al. Long-term results of stenting versus endarterectomy for carotid-artery stenosis. N Engl J Med 2016;374:1021-31.

13. Mas JL, Brown MM, Ringleb PA. Stenting versus endarterectomy for carotid-artery stenosis. N Engl J Med 2010;363:1766-7.

14. Bouri S, Thapar A, Shalhoub J, et al. Hypertension and the postcarotid endarterectomy cerebral hyperperfusion syndrome. Eur $J$ Vasc Endovasc Surg 2011;41:229-37.

15. Lin LM, Bender MT, Colby GP, et al. Use of a next-generation multi-durometer long guide sheath for triaxial access in flow diversion: experience in 95 consecutive cases. $J$ Neurointerv Surg 2018;10.

16. Pierce DS, Rosero EB, Modrall JG, et al. Open-cell versus closed-cell stent design differences in blood flow velocities after carotid stenting. J Vasc Surg 2009;49:602-6. 